$\underline{\text { Preprint typeset in JHEP style - PAPER VERSION }}$

\title{
Fluctuating Fuzzy Funnels
}

\author{
Rajsekhar Bhattacharyya ${ }^{2}$ and Robert de Mello Koch ${ }^{1,2}$, \\ Department of Physics and Centre for Theoretical Physics ${ }^{1}$, \\ University of the Witwatersrand, \\ Wits, 2050, \\ South Africa \\ Stellenbosch Institute for Advanced Studies, ${ }^{2}$ \\ Stellenbosch, \\ South Africa \\ E-mail: rbhattac@sun.ac.za, robert@neo.phys.wits.ac.za
}

\begin{abstract}
It is well known that a D-string ending on a D3, D5 or D7 brane is described in terms of a non-commutative fuzzy funnel geometry. In this article, we give a numerical study of the fluctuations about this leading geometry. This allows us to investigate issues related to the stability and moduli space of these solutions. We comment on the comparison to the linearized fluctuations in supergravity.
\end{abstract}

Keywords: Non-commutative geometry, D-branes, Fuzzy funnels. 


\section{Contents}

1. Introduction $\quad 1$

2. Review of the Fuzzy Funnel Solutions 3

2.1 Fuzzy Spheres 4

2.2 D1/D5 System 5

2.3 D1/D7 System 6

3. Parametrizing the Fluctuations $\quad 6$

3.1 D1/D5 System 8

3.2 D1/D7 System 9

$\begin{array}{ll}\text { 4. Problem Formulation } & 9\end{array}$

5. Results for the D1 $\perp$ D5 System 12

$5.1 n=1 \quad 12$

$5.2 n=2 \quad 13$

6. Comparison to Supergravity $\quad 15$

6.1 Linearized Born-Infeld $\quad 15$

6.2 Supergravity Analysis $\quad 16$

6.3 Newtonian Approximation 17

$\begin{array}{ll}\text { 7. Summary } & 20\end{array}$

\section{Introduction}

Fuzzy spheres are fascinating examples of non-commutative geometry. They have obvious application in string theory, where they arise as solutions to matrix brane actions[1][2][3][4][5], and may also play a role in a spacetime explanation of the stringy exclusion principle[6]. In this article we will be interested in describing small fluctuations about the so-called "fuzzy funnel" solutions describing D-strings ending on D3[1], D5[2] or D7[4] branes. The funnels have the topology $R \times S^{k}$ with $S^{k}$ a fuzzy sphere. 
Although all of the funnels that we consider are time independent, the equations for the funnel also admit interesting time dependent solutions[7].

An important difference between a point particle and an extended object is the fact that an extended object has a set of low energy excitations which arise from small vibrations about their equilibrium configuration. These modes have already played an important role in the study of black holes in string theory where they account for both the microscopic entropy[8] and the low energy Hawking radiation of the black holes[9]. There are a number of reasons why a study of the spectrum of small fluctuations of the fuzzy funnels is interesting. First, the prescence of tachyonic modes signals an instability in the solution. The fuzzy funnel relevant for D-strings ending on D7-branes has been given a description from both the point of view of the D-strings and of the D7branes[4]. It turns out that the two descriptions are in good agreement, at leading order in $N$. From the point of view of the D7 brane description, the funnel is described by a $7+1$ dimensional non-Abelian Born-Infeld theory with a specific non-zero field strength switched on. It appears[4] that it is possible to lower the energy of the funnel solution, by changing this field strength. Thus there are good reasons to question the stability of the D1 $\perp$ D7 funnel. Our analysis shows that the D1 $\perp$ D7 system is perturbatively stable. Second, if there is a family of funnel solutions this will show up as zero modes in the small fluctuation spectrum. Third, the geometry of the fuzzy funnel involves a fuzzy sphere. For the fuzzy $S^{4}$ and $S^{6}$ there are new degrees of freedom[10][11] that have no counterpart in the commutative $S^{4}$ and $S^{6}$. These degrees of freedom are most important at finite $N$ corresponding to situations in which quantum gravity corrections can not be neglected. Understanding the role of these new degrees of freedom is an important problem. By studying the small fluctuations of the funnel, we will gain some insight into these degrees of freedom.

The fluctuations with which we concern ourselves arise from the motion of the branes in spacetime. Consequently we do not consider the fermions or the gauge field that resides on the branes worldvolume. To carry out the small fluctuation analysis, we need a basis in which to expand our fluctuations. If we were dealing with the geometry of the usual commutative sphere, we would simply use the spherical harmonics. Since we deal with the fuzzy sphere we will have to use a deformed algebra, where the deformation allows for a cut off on the angular momentum. We will see that the usual approach to constructing this basis for the fluctuations is frustrated by the noncommuting nature of the coordinates. The natural guess - to take traceless symmetric combinations of the underlying coordinates - does not lead to a closed algebra. Fortunately, Ramgoolam[10] has found an elegant solution to this problem. For the $S^{2 n}$, instead of simply restricting to the traceless symmetric representations, one considers all possible representations of $S O(2 n+1)$, which may conveniently be labelled by Young 
Diagrams. The cut off on angular momentum is implemented as a cut off on the number of columns of the Young diagrams. Since there are only a finite number of independent basis functions in the deformed algebra the problem lends itself to a numerical study. In this article we provide this numerical analysis of the small fluctuations for both the $\mathrm{D} 1 \perp \mathrm{D} 5$ and the $\mathrm{D} 1 \perp \mathrm{D} 7$ systems.

In [2] small fluctuations of the D1 $\perp$ D 5 system were studied. The funnel analysis was performed where the funnel is narrow and hence it should be reliably described in terms of $N$ D-strings. These results did not match results obtained for a test-string in the background of $n$ D5 branes. Since both the D-string and the test string descriptions should be valid for large $\sigma$ this disagreement is rather disturbing. One of our goals in this article is to resolve this puzzle. Using a Newtonian approximation we are able to produce a model which uses the full funnel geometry as opposed to [2] where the funnel was effectively replaced by $n$ D5 branes. We then find that the dynamics of the fluctuations depends sensitively on the size of the fluctuation. Further, we show that it is possible to reproduce the funnel results using our Newtonian toy model.

In the next section we briefly review the fuzzy funnel solutions for both the D1 $\perp$ D 5 and the $\mathrm{D} 1 \perp \mathrm{D} 7$ systems. In section 3 we discuss in detail how a basis for the fluctuations can be constructed, allowing for a numerical solution of the small fluctuations problem. In section 4, we describe our formulation of the small fluctuations problem and in section 5 we give the results of this analysis. The analysis of sections 4 and 5 is only valid for small field values. In section 6 we compare our results to a gravity analysis. In section 7 we present our conclusions and indicate some directions in which this work can be extended.

\section{Review of the Fuzzy Funnel Solutions}

As mentioned above, the fuzzy funnel solutions are relevant for the description of Dstrings ending on either D3, D5 or D7 branes. The funnels have the topology $R \times S^{k}$ with $S^{k}$ a fuzzy sphere with $k=2$ for the D1 $\perp$ D3 system, $k=4$ for the D1 $\perp$ D 5 system and $k=6$ for the $\mathrm{D} 1 \perp \mathrm{D} 7$ system. We do not concentrate much on the $\mathrm{D} 1 \perp \mathrm{D} 3$ system in this article. The fuzzy funnels can be described using either a worldvolume description of the strings, or a worldvolume description of the branes. The two descriptions are in perfect agreement. We will focus on the description in terms of the D-strings.

The low energy effective action for $N$ D-strings is given by the non-Abelian BornInfeld action[12],[13] 


$$
S=-T_{1} \int d^{2} \sigma S \operatorname{Tr} \sqrt{-\operatorname{det}\left[\begin{array}{cc}
\eta_{a b} & \lambda \partial_{a} \Phi^{j} \\
-\lambda \partial_{b} \Phi^{i} & Q^{i j}
\end{array}\right]} \equiv-T_{1} \int d^{2} \sigma S \operatorname{Tr} \sqrt{-\operatorname{det} M}
$$

where

$$
Q^{i j}=\delta^{i j}+i \lambda\left[\Phi^{i}, \Phi^{j}\right], \quad \lambda=2 \pi l_{s}^{2}
$$

The symmetrized trace prescription [14] (indicated by $S T r$ in the above action) instructs us to symmetrize over all permutations of $\partial_{a} \Phi^{i}$ and $\left[\Phi^{i}, \Phi^{j}\right]$ within the trace over the gauge group indices, after expanding the square root. We are using static gauge so that the worldsheet coordinates are identified with spacetime coordinates as $\tau=x^{0}$ and $\sigma=x^{9}$. The transverse coordinates are now the non-Abelian scalars $\Phi^{i}$, $i=1, \ldots, 8$. These scalars are $N \times N$ matrices transforming in the adjoint representation of the $U(N)$ gauge symmetry of the worldsheet theory of the D1s.

The lowest order equation of motion is

$$
\left(-\partial_{\tau}^{2}+\partial_{\sigma}^{2}\right) \Phi^{i}=\left[\Phi^{j},\left[\Phi^{j}, \Phi^{i}\right]\right]
$$

This equation of motion is valid for small $\Phi^{j}$, where the funnel is accurately described by a collection of D1s. We are interested in studying the fluctuations $\delta \Phi^{i}$ about the leading configuration $\Phi_{0}^{i}$

$$
\Phi^{i}=\Phi_{0}^{i}+\delta \Phi^{i}
$$

The linearized equation for the fluctuation following from this lowest order equation of motion is

$$
\left(-\partial_{\tau}^{2}+\partial_{\sigma}^{2}\right) \delta \Phi^{i}=\left[\delta \Phi^{j},\left[\Phi_{0}^{j}, \Phi_{0}^{i}\right]\right]+\left[\Phi_{0}^{j},\left[\delta \Phi^{j}, \Phi_{0}^{i}\right]\right]+\left[\Phi_{0}^{j},\left[\Phi_{0}^{j}, \delta \Phi^{i}\right]\right] .
$$

It is this last equation that we analyze in detail in sections 4 and 5.

In the remainder of this section, we give the leading configurations $\Phi_{0}^{i}$. We start by briefly reviewing the construction of fuzzy spheres, and then discuss the funnels themselves. In the next section, we will provide a basis[10] in terms of which the fluctuations $\delta \Phi^{i}$ can be expanded.

\subsection{Fuzzy Spheres}

To construct the fuzzy $S^{2 m}$ sphere, we need to solve the equation 


$$
\sum_{i=1}^{2 m+1} X^{i} X^{i}=c \mathbf{1}
$$

with $X^{i}$ a matrix, 1 the identity matrix and $c$ a constant. There is a simple construction of the matrices $X^{i}$, in terms of the Clifford algebra

$$
\left\{\Gamma^{i}, \Gamma^{j}\right\}=2 \delta^{i j}, \quad i, j=1,2, \ldots, 2 m+1 .
$$

The $n$-fold tensor product of $V$ (the space on which the $\Gamma^{i}$ matrices act) is written as $V^{\otimes n}$. The $X^{i}$ are now obtained by taking

$$
X^{i}=\left(\Gamma^{i} \otimes 1 \otimes \ldots \otimes 1+1 \otimes \Gamma^{i} \otimes \ldots \otimes 1+\ldots+1 \otimes 1 \otimes \ldots \otimes \Gamma^{i}\right)_{s t} .
$$

The subscript st is to indicate that the above $X^{i}$ are to be restricted to the completely symmetric tensor product space for $2 m=2,4$, and symmetric and traceless tensor product space for $2 m=6$. Clearly, the $X^{i}$ act in $V^{\otimes n}$. To prove that the above $X^{i}$ do indeed provide coordinates for the fuzzy sphere, one shows that $\sum_{i=1}^{2 m+1} X^{i} X^{i}$ commutes with the generators of $S O(2 m+1)$; then by Schur's lemma, it follows that $\sum_{i=1}^{2 m+1} X^{i} X^{i}$ is proportional to the identity.

For properties of the matrices $X^{i}$ in the case of the fuzzy $S^{4}$, see [3] and in the case of the fuzzy $S^{6}$, see [15]

\subsection{D1/D5 System}

We use $N$ to denote the number of D-strings in the funnel and $n$ to denote the number of D5-branes in the funnel. Five of the eight scalars are excited

$$
\Phi_{0}^{i}=R(\sigma) X^{i}, \quad i=1,2,3,4,5,
$$

where the function $R(\sigma)$ is, for small $R$,

$$
R(\sigma)=\frac{1}{2 \sqrt{2} \sigma}
$$

In this case, the matrices $X^{i}$ have dimension

$$
N=\frac{1}{6}(n+1)(n+2)(n+3),
$$

and $c=n(n+4)$. 


\subsection{D1/D7 System}

We use $N$ to denote the number of D-strings in the funnel and $n$ to denote the number of D7-branes in the funnel. Seven of the eight scalars are excited

$$
\Phi_{0}^{i}=R(\sigma) X^{i}, \quad i=1,2, \ldots, 7,
$$

where the function $R(\sigma)$ is, for small $R$,

$$
R(\sigma)=\frac{1}{2 \sqrt{3} \sigma} .
$$

In this case, the matrices $X^{i}$ have dimension

$$
N=\frac{1}{360}(n+1)(n+2)(n+3)^{2}(n+4)(n+5),
$$

and $c=n(n+6)$.

\section{Parametrizing the Fluctuations}

To study the geometry of $S^{2}$ embedded in $R^{3}$, we can study the algebra of smooth functions over $S^{2}$. This algebra can be taken as a commutative polynomial algebra, i.e the polynomial ring $R\left[x^{1}, x^{2}, x^{3}\right]$ together with the constraint

$$
\left(x^{1}\right)^{2}+\left(x^{2}\right)^{2}+\left(x^{3}\right)^{2}=c .
$$

In other words we can study $R\left[x^{1}, x^{2}, x^{3}\right] / I$ where $I$ is the ideal generated by the polynomial $\left(x^{1}\right)^{2}+\left(x^{2}\right)^{2}+\left(x^{3}\right)^{2}-c$.

It is then natural to study the geometry of the fuzzy $S^{2}$ by studying the algebra of functions over it. For some fixed positive integer $n$ we replace $x^{1}, x^{2}, x^{3}$ by three $n \times n$ matrices $X^{1}, X^{2}, X^{3}$, which generate the $S U(2)$ Lie algebra

$$
\left[X^{i}, X^{j}\right]=i K C^{i j k} X^{k},
$$

where $C^{i j k}=\epsilon^{i j k}$ and where $K$ depends on the integer $n$. We still need to impose the analog of the constraint (3.1). It turns out that there is a one-to-one correspondence between the constraints in the case of the commutative $S^{2}$ and the constraints in the case of the fuzzy $S^{2}$. This one-to-one correspondence between the constraints can be traced back to the fact that the $X^{i}$ 's form a closed Lie algebra among themselves. Thus, the basis elements for the non-commutative algebra over the fuzzy $S^{2}$, can be obtained by replacing $x^{i} \rightarrow X^{i}$ in the basis elements of the algebra for the commutative $S^{2}$. Note however that we have an upper bound on the number of basis elements in 
the algebra over the fuzzy $S^{2}$, depending on the dimension of the representation of the $S U(2)$ Lie algebra.

For the case of $S^{4}$ and fuzzy $S^{4}$ or $S^{6}$ and fuzzy $S^{6}$ things are not so simple. We discuss in detail the case of the fuzzy $S^{4}$. For the geometry of the commutative $S^{4}$, the algebra of functions is given by $R\left[x^{1}, x^{2}, x^{3}, x^{4}, x^{5}\right] / I^{\prime}$ where $I^{\prime}$ is the ideal generated by the polynomial $\left(x^{1}\right)^{2}+\left(x^{2}\right)^{2}+\left(x^{3}\right)^{2}+\left(x^{4}\right)^{2}+\left(x^{5}\right)^{2}-c$. For the fuzzy $S^{4}$, we replace $x^{i}$ 's by the $X^{i}$ 's given above. The $X^{i}$ 's do not form a closed Lie algebra. They can be extended to the $S O(5,1)$ Lie algebra by including the new generators

$$
X^{i j}=\frac{1}{2}\left[X^{i}, X^{j}\right]
$$

The need to include these new elements implies that there are some constraints that do not follow from the two sided ideal generated by

$$
\left(X^{1}\right)^{2}+\left(X^{2}\right)^{2}+\left(X^{3}\right)^{2}+\left(X^{4}\right)^{2}+\left(X^{5}\right)^{2}-c I .
$$

To illustrate this point, note that the relation[2]

$$
X^{i j} X^{j}-X^{j} X^{j i}=0
$$

follows from

$$
\begin{array}{r}
\quad \frac{1}{2} X^{i}\left(\left(X^{1}\right)^{2}+\left(X^{2}\right)^{2}+\left(X^{3}\right)^{2}+\left(X^{4}\right)^{2}+\left(X^{5}\right)^{2}-c I\right) \\
-\frac{1}{2}\left(\left(X^{1}\right)^{2}+\left(X^{2}\right)^{2}+\left(X^{3}\right)^{2}+\left(X^{4}\right)^{2}+\left(X^{5}\right)^{2}-c I\right) X^{i}
\end{array}
$$

so that it can be written as an element of the two sided ideal; in contrast, the relation

$$
X^{i j} X^{j}-4 X^{i}=0
$$

only follows after using the $S O(5,1)$ Lie algebra. This spoils any possible connection between the basis functions in the commutative and non-commutative cases. Thus, finding a suitable basis in which to expand the fluctuations of the fuzzy $S^{4}$ and fuzzy $S^{6}$ can not simply be obtained by replacing $x^{i} \rightarrow X^{i}$ in the basis for the commutative $S^{4}$ and $S^{6}$.

Fortunately, Ramgoolam[10] has given an elegant solution to this problem. In the remainder of this section, we describe the basis provided by[10] for both the fuzzy $S^{4}$ (which is relevant for the D1 $\perp$ D5 system) and for the fuzzy $S^{6}$ (which is relevant for the $\mathrm{D} 1 \perp \mathrm{D} 7$ system). In particular, we will describe our algorithm for generating the basis that was used in the numerical computation of the spectrum. 


\subsection{D1/D5 System}

In this section we will describe an $S O(5)$ covariant basis for the $N \times N$ matrices $\delta \Phi^{i}$. Elements of this basis are labelled by irreducible representations of $S O(5)$ which are themselves associated with a unique Young diagram. The Young diagrams labelling the representations have at most two rows. To translate a specific Young diagram into a basis element, we associate a set of indices to the Young diagram. For example, consider the representation associated with the Young diagram with two boxes in the first row and one box in the second row. A suitable set of indices would be

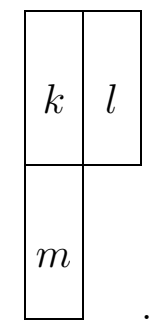

Each index is associated with an $X^{i}$; for the above example, the basis element is built from $X^{k}, X^{l}$ and $X^{m}$. The shape of the Young diagram gives a rule for how the $X^{i} \mathrm{~s}$ should be combined: First we symmetrize over indices in the same row

$$
X^{k} X^{l} X^{m} \rightarrow\left(X^{k} X^{l}+X^{l} X^{k}\right) X^{m}=X^{k} X^{l} X^{m}+X^{l} X^{k} X^{m},
$$

and then antisymmetrize over indices in the same column

$$
X^{k} X^{l} X^{m}+X^{l} X^{k} X^{m} \rightarrow X^{k} X^{l} X^{m}-X^{m} X^{l} X^{k}+X^{l} X^{k} X^{m}-X^{l} X^{m} X^{k} .
$$

Numerically, it is straightforward to implement this symmetrization and then the antisymmetrization. There is an additional complication because we deal with representations of the orthogonal group: the above state can be written as

$$
X^{k} X^{l} X^{m}-X^{m} X^{l} X^{k}+X^{l} X^{k} X^{m}-X^{l} X^{m} X^{k} \equiv C_{a b c} X^{a} X^{b} X^{c} .
$$

To get a state which belongs to the advertised irreducible representation, we need to restrict ourselves not only to tensors $C_{a b c}$ with the correct symmetrization and antisymmetrization properties, but also, tensors that are traceless. Numerically we implemented this traceless constraint by subtracting out any projections our basis function had on irreducible representations corresponding to Young diagrams containing a smaller number of boxes. The cut off on angular momentum becomes a cut off on the number of columns in the Young diagrams. One can show[10] that the number of 
independent states in the irreducible representations included by this prescription is precisely equal to

$$
N^{2}=\frac{(n+1)^{2}(n+2)^{2}(n+3)^{2}}{36},
$$

and hence that we do indeed obtain a complete basis in which to express the $\delta \Phi^{i}$.

Numerically, we performed an exhaustive search over all possible placements of indices in the allowed Young diagrams. Of course, many of the operators constructed in this way were identically zero. Further, not all of the operators constructed were independent, due to the tracelessness constraint. Only the independent operators were kept. Our numerical results show clearly that one does indeed construct a complete orthonormal basis using the procedure outlined in [10].

\subsection{D1/D7 System}

In this case, one sums over irreducible representations of $S O(7)$ which can be labelled by Young diagrams with three rows. Again the cut off is implemented on the columns of the Young diagram. In this case, one expects a total of[10]

$$
N^{2}=\frac{(n+1)^{2}(n+2)^{2}(n+3)^{4}(n+4)^{2}(n+5)^{2}}{129600}
$$

basis functions. Again, our numerical results are in perfect agreement with this expectation.

\section{Problem Formulation}

We are now ready to compute the spectrum of the small fluctuations. As discussed in section 2, we consider the lowest order equation of motion. Thus our results will only be valid for small $\Phi_{0}^{i}$, corresponding to the large $\sigma$ region of both funnels. In this limit both funnels look like a collection of D-strings.

The problem of computing the small fluctuations essentially reduces to solving the eigenvalue problem for the operator $L$ defined by $(i=1, \ldots, 8)$

$$
L v^{(C)}(i)=\left[v^{(C)}(j),\left[\phi^{j}, \phi^{i}\right]\right]+\left[\phi^{j},\left[v^{(C)}(j), \phi^{i}\right]\right]+\left[\phi^{j},\left[\phi^{j},\left[v^{(C)}(i)\right]\right]=\lambda(C) v^{(C)}(i) .\right.
$$

The fluctuation is labelled by $C$. The index $i$ labels the components of a particular fluctuation. Thus $v^{(C)}(i)$, fluctuation $C$, is to be viewed as a vector with 8 components; each component is an $N \times N$ matrix. Denote the basis elements described in the previous section by $\beta^{A}$, with $A=1, \ldots, N^{2}$. Further, introduce a basis for the fluctuations, given 
by $|C\rangle=|\{A, i\}\rangle$. All of the components of the vector $|\{A, i\}\rangle$ are the $N \times N$ zero matrix, except the $\mathrm{i}^{\text {th }}$ component, which is $\beta^{A}$. Thanks to the fact that $L$ has a linear action on $v^{(C)}$, the eigenvectors of $L$ can be expressed as a linear combination of the $|\{A, i\}\rangle$. Writing

$$
|\{A, i\}\rangle=\left[\begin{array}{c}
\beta^{A} \delta^{i 1} \\
\beta^{A} \delta^{i 2} \\
: \\
\beta^{A} \delta^{i 8}
\end{array}\right]
$$

we define the inner product

$$
\left\langle\{A, i\} \mid\left\{A^{\prime}, i^{\prime}\right\}\right\rangle \equiv \delta^{i i^{\prime}} \operatorname{Tr}\left(\beta^{A \dagger} \beta^{A^{\prime}}\right) .
$$

We will normalize our states so that $\langle\{B, j\} \mid\{A, i\}\rangle=\delta^{A B} \delta^{i j}$. Using the completeness relation

$$
\sum_{A, i}|\{A, i\}\rangle\langle\{A, i\}|=\mathbf{1}
$$

where $\mathbf{1}$ is the $8 N^{2} \times 8 N^{2}$ identity matrix, we can write

$$
L|\{A, i\}\rangle=\sum_{B, j}\langle\{B, j\}|L|\{A, i\}\rangle|\{B, j\}\rangle,
$$

where $\langle\{B, j\}|L|\{A, i\}\rangle$ is an $8 N^{2} \times 8 N^{2}$ matrix. In terms of the eigenvectors of this matrix

$$
\sum_{A, i}\langle\{B, j\}|L|\{A, i\}\rangle u^{(C)}(A, i)=\lambda(C) u^{(C)}(B, j),
$$

the eigenvectors of $L$ can be written as

$$
v^{(C)}=\sum_{A, i} u^{(C)}(A, i)|\{A, i\}\rangle
$$

Our approach entails computing the matrix $\langle B, j|L| A, i\rangle$ and diagonalizing it numerically to obtain $\lambda(C)$. After making the ansatz

$$
\delta \Phi^{i}=f^{(C)}(\sigma, \tau) v^{(C)}(i)
$$

the lowest order equation of motion then reduces to

$$
\left(-\partial_{\tau}^{2}+\partial_{\sigma}^{2}-\lambda(C) R^{2}(\sigma)\right) f^{(C)}(\sigma, \tau)=0
$$


which can be dealt with analytically.

Zero modes (modes with $\lambda(C)=0$ ) correspond to massless fluctuations on the worldvolume theory of the $\mathrm{D}$-string. It is natural to interpret these modes as Goldstone bosons associated with the spontaneous breaking of translation, rotation and boost invariance of the worldvolume theory of the brane. In the large $\sigma$ region where we can use (for the $\mathrm{D} 1 \perp \mathrm{D} 3$ system this is true for all $\sigma$ )

$$
R(\sigma) \propto \frac{1}{\sigma}
$$

we find that $f(\sigma, \tau)$ satsifies

$$
\left(-\partial_{\tau}^{2}+\partial_{\sigma}^{2}-\frac{\rho}{\sigma^{2}}\right) f^{(C)}(\sigma, \tau)=0 .
$$

Modes with $\lambda(C)<0$ correspond to modes with $\rho<0$. Making the ansatz $f^{(C)}(\sigma, \tau)=$ $e^{i \omega \tau} g(\sigma)$, we find the following eigenvalue problem

$$
\omega^{2} g(\sigma)=\left(-\partial_{\sigma}^{2}-\frac{|\rho|}{\sigma^{2}}\right) g(\sigma) \equiv H g(\sigma) .
$$

The eigenvalue problem for this quantum mechanical Hamiltonian has been studied previously; recent results include [16],[17]. We will review an argument given in [16] which provides a simple illustration of the instability of the D1 $\perp$ D3 funnel for $\lambda(C)<0$. Consider

$$
\frac{\langle\psi|H| \psi\rangle}{\langle\psi \mid \psi\rangle}=\frac{\int\left|\psi^{\prime}(\sigma)\right|^{2} d \sigma-|\rho| \int \frac{|\psi(\sigma)|^{2}}{\sigma^{2}} d \sigma}{\int|\psi(\sigma)|^{2} d \sigma} .
$$

Set $\psi_{\lambda}(\sigma)=\sqrt{\lambda} \psi(\lambda \sigma)$ so that

$$
\frac{\left\langle\psi_{\lambda}|H| \psi_{\lambda}\right\rangle}{\left\langle\psi_{\lambda} \mid \psi_{\lambda}\right\rangle}=\lambda^{2} \frac{\int\left|\psi^{\prime}(\sigma)\right|^{2} d \sigma-|\rho| \int \frac{|\psi(\sigma)|^{2}}{\sigma^{2}} d \sigma}{\int|\psi(\sigma)|^{2} d \sigma} .
$$

This demonstrates that if[16]

$$
|\rho|>\frac{\int\left|\psi^{\prime}(\sigma)\right|^{2} d \sigma}{\int \frac{|\psi(\sigma)|^{2}}{\sigma^{2}} d \sigma}
$$

then $H$ is unbounded from below indicating an infinite number of negative energy states. It is possible to argue further that[16] this will occur when $|\rho|>\frac{1}{4}$.

The existence of negative energy states (i.e. the existence of bound states for the above Hamiltonian) implies that we have modes with $\omega^{2}<0$ and which are thus tachyonic. For a large enough negative eigenvalue we see that the D1 $\perp$ D3 system has 
an infinite number of tachyonic modes. Thus, instabilities of the $\mathrm{D} 1 \perp \mathrm{D} 3$ funnel show up as negative eigenvalues $\lambda(C)^{1}$.

These results do not immediately generalize to the $\mathrm{D} 1 \perp \mathrm{D} 5$ and the $\mathrm{D} 1 \perp \mathrm{D} 7$ funnels, because the bound states have their support at small $\sigma$ which is precisely the region in which the funnels differ. For example, the Hamiltonian relevant for the D1 $\perp$ D 5 funnel, for small $\sigma$ is

$$
\omega^{2} g(\sigma)=\left(-\partial_{\sigma}^{2}-\frac{|\rho|}{\sigma^{2 / 3}}\right) g(\sigma) \equiv H g(\sigma) .
$$

Although this potential is not as "steep" as for the D1 $\perp$ D3 system, we still expect bound states (signalling tachyons) for large enough $|\rho|$. Modes with $\lambda(C)>0$ correspond to a repulsive potential and hence there can be no bound states.

Thus, in conclusion modes with $\lambda(C)<0$ need to be considered carefully as they may indeed signal instabilities.

\section{Results for the D1 $\perp$ D5 System}

The size of the matrix $\langle\{B, j\}|L|\{A, i\}\rangle$ grows rapidly with $n$. For $n=1$ there are a total of 16 basis elements and the matrix $\langle\{B, j\}|L|\{A, i\}\rangle$ is a $128 \times 128$ dimensional matrix; for $n=2$ there are a total of 100 basis elements and the matrix $\langle\{B, j\}|L|\{A, i\}\rangle$ is an $800 \times 800$ dimensional matrix; for $n=3,\langle\{B, j\}|L|\{A, i\}\rangle$ is a $3200 \times 3200$ dimensional matrix. In this section we will discuss the results obtained for $n=1,2$.

\section{$5.1 n=1$}

If we had been working on a commutative sphere, then modes with an angular momentum less than or equal to 1 would be expressed in terms of the scalar or the $l=1$ partial waves. Here the scalar mode is replaced by the identity matrix and the first partial waves by the $X^{i}$. We call fluctuations that can be expressed in terms of the identity and $X^{i}$ the classical contribution. Since the coordinates do not commute we also have contributions that are expressed in terms of $\left[X^{k}, X^{l}\right]$. These have no analog in the case of a commutative geometry and hence we refer to these fluctuations as the quantum contribution. At this order and at $n=1$, to obtain the full set of fluctuations, we have checked that there is no need to mix the new quantum degrees of freedom and the degrees of freedom that would be present for a commutative sphere. However, we expect this to change once interactions are included and have verified that there is mixing for

\footnotetext{
${ }^{1}$ The eigenvalues $\lambda(C)$ for the $\mathrm{D} 1 \perp \mathrm{D} 3$ system are in fact all positive, as expected, since the $\mathrm{D} 1 \perp \mathrm{D} 3$ system is supersymmetric and hence obviously stable.
} 
$n>1$. Further, looking at the eigenvalues of the fluctuations, we see that the dynamics does not discriminate against the new degrees of freedom; they have eigenvalues that are comparable to the eigenvalues of the degrees of freedom that would be present for a commutative sphere. The spectrum of eigenvalues is

$\begin{array}{cccc}\text { Eigenvalue } & \text { Degeneracy } & \text { Classical Contribution } & \text { Quantum Contribution } \\ 0 & 43 & 8 & 35 \\ 8 & 44 & 14 & 30 \\ 16 & 30 & 25 & 5 \\ 24 & 10 & 0 & 10 \\ 48 & 1 & 1 & 0\end{array}$

Since there are no negative eigenvalues, we see that to the order we are working, the D1/D5 funnel is stable.

The modes with eigenvalue equal to zero are giving us information about the moduli space (that is, the space of solutions) of the fuzzy sphere. Intuition from the commutative case suggests that this moduli space is generated by performing translations and $S O(1,5)$ transformations on the fivebrane worldvolume. Looking at the above results, we find that there are 8 zero modes corresponding to translations of the $X^{i}$

$$
\delta \Phi^{i} \propto 1
$$

The $S O(1,5)$ transformations would include 5 boosts and 10 rotations (symmetries of $\left.S^{4}\right)$. However, we have a remaining 35 zero modes that are all excitations involving only the new quantum degrees of freedom $\left[X^{i}, X^{j}\right]$. It has already been argued [10],[11] that the non-commutative $S^{4}$ can be viewed as a fuzzy $S^{2}$ fiber over an $S^{4}$ base. The symmetry of a commutative $S^{2}$ is $S O(3)$ and of a commutative $S^{4}$ is $S O(5)$. Assuming the symmetries acting on the base and fiber act independently one might expect that the 10 rotations are replaced by $3 \times 10=30$ rotations when we consider the noncommutative $S^{4}$. Thus, it seems natural to expect 30 rotations and 5 boosts giving 35 zero modes for the fuzzy funnel in complete agreement with what we have obtained numerically. If this interpretation is correct, we see that the zero mode spectrum reflects, in a rather transparent way, the extra dimensions present in the fuzzy sphere geometries[10],[11].

\section{$5.2 n=2$}

In this case the generic fluctuation is a linear combination of the new quantum degrees of freedom and the degrees of freedom that would be present for a commutative sphere. The spectrum of eigenvalues is 


$\begin{array}{cc}\text { Eigenvalue } & \text { Degeneracy } \\ 0 & 148 \\ 8 & 44 \\ 16 & 300 \\ 24 & 40 \\ 32 & 140 \\ 40 & 42 \\ 48 & 71 \\ 72 & 10 \\ 80 & 5\end{array}$

Again, the absence of negative eigenvalues implies that the solution is stable.

We again find 8 zero modes corresponding to translations of the $X^{i}$. The interpretation of the remaining 140 zero modes does not seem to be so simple. Assuming our interpretation for the zero modes given above is correct, the fact that $140-5=135$ is not a multiple of 30 suggests that the symmetries acting on the base and fiber no longer act independently. Clearly, clarifying the connection between the zero modes and symmetries of the non-commuative geometry in general remains an interesting open problem.

\section{Results for the D1 $\perp$ D7 System}

We will only consider the case $n=1$. There are 64 basis elements and the matrix $\langle\{B, j\}|L|\{A, i\}\rangle$ is a $512 \times 512$ dimensional matrix. In this case, it is again possible to classify the small fluctuations as classical or quantum. Here the quantum contributions include both $\left[X^{i}, X^{j}\right]$ and

$$
X^{i j k}=X^{i} X^{j} X^{k}+X^{j} X^{k} X^{i}+X^{k} X^{i} X^{j}-X^{i} X^{k} X^{j}-X^{j} X^{i} X^{k}-X^{k} X^{j} X^{i} .
$$

This is a direct consequence of the fact that the Young diagrams for $S O(7)$ have three rows. The spectrum of eigenvalues is 


$\begin{array}{cccc}\text { Eigenvalue } & \text { Degeneracy } & \text { Classical Contribution } & \text { Quantum Contribution } \\ 0 & 113 & 8 & 105 \\ 8 & 210 & 0 & 210 \\ 16 & 62 & 27 & 35 \\ 24 & 105 & 28 & 77 \\ 48 & 21 & 0 & 21 \\ 72 & 1 & 1 & 0\end{array}$

The absence of negative eigenvalues suggests that this solution is perturbatively stable. There are 113 zero modes. We expect 8 of these zero modes correspond to translations. The $S O(1,7)$ symmetry of the worldvolume theory of the D7 branes includes 7 boosts. The remaining 98 zero modes are presumably associated with symmetries of the fuzzy $S^{6}$.

\section{Comparison to Supergravity}

In this section we study the linearized equations for the full Born-Infeld action. For the $\mathrm{D} 3 \perp \mathrm{D} 1$ system, these linearized equations correctly reproduce some of the modifications of the supergravity background generated by the D3 branes[18],[19],[1]. For the $\mathrm{D} 5 \perp \mathrm{D} 1$ system a test-string in the background generated by $n$ D5-branes does not agree with the linearized equation describing small fluctuations of the D1 $\perp$ D5 funnel[2]. An understanding of this discrepancy is required for a proper interpretation of the fuzzy funnel.

\subsection{Linearized Born-Infeld}

Following[2] we consider the fluctuation

$$
\delta \Phi^{6}(\sigma, \tau)=f(\sigma, \tau) \mathbf{1}
$$

with 1 the $N \times N$ identity matrix. Plugging this into the D-string action (in the background of the funnel $\left.\Phi_{0}^{i}=R G^{i} / \sqrt{c} \lambda\right)$ we obtain

$$
\begin{aligned}
S & =-N T_{1} \int d^{2} \sigma \sqrt{\left(1+R^{\prime 2}\right)\left(1-\lambda^{2} \dot{f}^{2}\right)+\lambda^{2} f^{\prime 2}}\left[1+4 R^{4} /\left(c \lambda^{2}\right)\right] \\
& =-N T_{1} \int d^{2} \sigma\left(H-\frac{1}{2} \lambda^{2} H \dot{f}^{2}+\frac{1}{2} f^{\prime 2}\right) \quad H(\sigma)=\left(1+\frac{4 R(\sigma)}{c \lambda^{2}}\right)^{2} .
\end{aligned}
$$

Only the quadratic terms are kept as these determine the linearized equation of motion 


$$
0=\left(H \partial_{\tau}^{2}-\partial_{\sigma}^{2}\right) f(\tau, \sigma) \approx\left(\left[1+\frac{n^{2} \lambda^{2}}{8 \sigma^{4}}\right] \partial_{\tau}^{2}-\partial_{\sigma}^{2}\right) f(\tau, \sigma) .
$$

It is natural to ask about the dependence of this linearized equation of motion on the specific matrix structure assumed for the fluctuation we consider. Towards this end, note that to evaluate the D-string action, we need to compute the terms quadratic in $\delta \Phi^{6}$ in the determinant

$$
\operatorname{det}\left[\begin{array}{cccccccc}
-1 & 0 & 0 & 0 & 0 & 0 & 0 & \lambda \partial_{\tau} \delta \Phi^{6} \\
0 & 1 & \lambda \partial_{\sigma} \Phi^{1} & \lambda \partial_{\sigma} \Phi^{2} & \lambda \partial_{\sigma} \Phi^{3} & \lambda \partial_{\sigma} \Phi^{4} & \lambda \partial_{\sigma} \Phi^{5} & \lambda \partial_{\sigma} \delta \Phi^{6} \\
0 & -\lambda \partial_{\sigma} \Phi^{1} & 1 & Q^{12} & Q^{13} & Q^{14} & Q^{15} & Q^{16} \\
0 & -\lambda \partial_{\sigma} \Phi^{2} & Q^{21} & 1 & Q^{23} & Q^{24} & Q^{25} & Q^{26} \\
0 & -\lambda \partial_{\sigma} \Phi^{3} & Q^{31} & Q^{32} & 1 & Q^{34} & Q^{35} & Q^{36} \\
0 & -\lambda \partial_{\sigma} \Phi^{4} & Q^{41} & Q^{42} & Q^{43} & 1 & Q^{45} & Q^{46} \\
0 & -\lambda \partial_{\sigma} \Phi^{5} & Q^{51} & Q^{52} & Q^{53} & Q^{54} & 1 & Q^{56} \\
-\lambda \partial_{\tau} \delta \Phi^{6} & -\lambda \partial_{\sigma} \delta \Phi^{6} & Q^{61} & Q^{62} & Q^{63} & Q^{64} & Q^{65} & 1
\end{array}\right]
$$

It is easy to see that to $O\left(\left(\delta \Phi^{6}\right)^{2}\right)$, the $\sigma$ dependence of the coefficients of both $\left(\delta \dot{\Phi}^{6}\right)^{2}$ and $\left(\delta \Phi^{\prime 6}\right)^{2}$ are unchanged. New terms of the form $\left(\delta \Phi^{6}\right)^{2}$ and $\delta \Phi^{6} \delta \Phi^{\prime 6}$ may be generated. This is enough to see that the $\sigma$ dependence of the $\partial_{\tau}^{2} \delta \Phi^{6}$ and $\partial_{\sigma}^{2} \delta \Phi^{6}$ terms in the linearized equation of motion are independent of the specific matrix structure assumed for the fluctuation we consider.

\subsection{Supergravity Analysis}

The results of the previous section should correspond to the fluctuation equation for a test D-string in some supergravity background. Assuming a diagonal background metric the test D-string action is[2]

$$
S=-N T_{1} \int d^{2} \sigma\left(\sqrt{h}-\frac{1}{2} \lambda^{2} h^{3 / 2} \dot{f}^{2}+\frac{1}{2} \lambda^{2} \sqrt{h} f^{\prime 2}\right) .
$$

For $n$ D5 branes

$$
d s^{2}=h(\sigma)^{-\frac{1}{2}} \eta_{\mu \nu} d x^{\mu} d x^{\nu}+h(\sigma)^{-\frac{1}{2}}\left(d \sigma^{2}+\sigma^{2} d \Omega_{3}^{2}\right), \quad h(\sigma)=1+\frac{L^{2}}{\sigma^{2}},
$$

the resulting linearized equation of motion for $f$ can be written as[2]

$$
\left(h^{2} \partial_{\tau}^{2}-\partial_{\tilde{\sigma}}^{2}\right) f(\tilde{\sigma}, \tau)=0, \quad \tilde{\sigma}^{2}=\sigma^{2}+L^{2} .
$$


This does not match the result following from expansion of the Born-Infeld action, as first observed in [2]. Further, as argued in the previous section, this mismatch is independent of the specific matrix structure assumed for the fluctuation of the fuzzy funnel description. This disagreement is puzzling as both the D-string and test-string descriptions should be valid for large $\sigma$. The disagreement can be traced back to the fact that all three funnel solutions have the same large $\sigma$ behaviour, so that the fluctuations from all three funnels look similar to the $\mathrm{D} 3 \perp \mathrm{D} 1$ system.

\subsection{Newtonian Approximation}

In this section we will build a Newtonian gravity description of the funnel. Our analysis provides a toy model description of the full dynamics that illustrates some subtle points, explaining the mismatch between the supergravity and linearized Born-Infeld descriptions.

To compare the Newtonian and supergravity descriptions, recall the well known fact that for a particle moving with a small velocity, in a region of spacetime well approximated by a flat metric, the geodesic equation becomes (we take $\tau$ to be an affine parametrization)

$$
\frac{d^{2} x^{i}}{d \tau^{2}}=\Gamma_{\alpha \beta}^{i} \frac{d x^{\alpha}}{d \tau} \frac{d x^{\beta}}{d \tau} \approx \frac{1}{2} \frac{d g_{00}}{d x^{i}},
$$

so that we can compare $g_{00}$ to the gravitational potential computed in the Newtonian theory.

A segment of the funnel stretching from $\sigma$ to $\sigma+d \sigma$ has a mass $(d=2$ for the $\mathrm{D} 1 \perp \mathrm{D} 3$ system and $d=4$ for the $\mathrm{D} 1 \perp \mathrm{D} 5$ system)

$$
d m=T_{d+1} \sqrt{1+R^{\prime}(\sigma)} R^{d}(\sigma) \Omega_{d} d \sigma .
$$

In 9 spatial dimensions, the potential of a point mass drops off as $1 / r^{7}$. Thus, the potential set up by the funnel is

$$
\phi=\int \frac{T_{d+1} \sqrt{1+R^{\prime}(\sigma)} R^{d}(\sigma) \Omega_{d}}{r^{7}} d \sigma,
$$

where $r$ is the distance from the observation point $\left(\vec{x}_{o b s}\right)$ to the segment of the funnel stretching from $\sigma$ to $\sigma+d \sigma$

$$
\begin{gathered}
r=\left|\vec{x}_{o b s}-\vec{x}_{\text {funnel segment }}\right|, \\
\vec{x}_{\text {funnel segment }}=\left(x^{0}, \sigma, R(\sigma) \vec{\Omega}, 0, \overrightarrow{0}\right),
\end{gathered}
$$




$$
\vec{x}_{o b s}=\left(x^{0}, \sigma_{o}, \overrightarrow{0}, x_{o}^{d+2}, \overrightarrow{0}\right)
$$

In what follows we will discuss only the D1 $\perp$ D 5 system. We have checked that exactly the same behaviour is exhibited by the D1 $\perp$ D3 system. Consider the integrand in the expression for $\phi$. We will study this integrand as a function of $\sigma$ for different values of $x_{o}^{6}$. The behaviour we will discuss below is qualitatively independent of the details of $R(\sigma)$. For this reason we will take $R(\sigma)=\frac{1}{\sigma}$ to illustrate our arguments. We will set $\sigma_{o}=2$. For $x_{o}^{6}=2$, the integrand is shown in figure 1 .

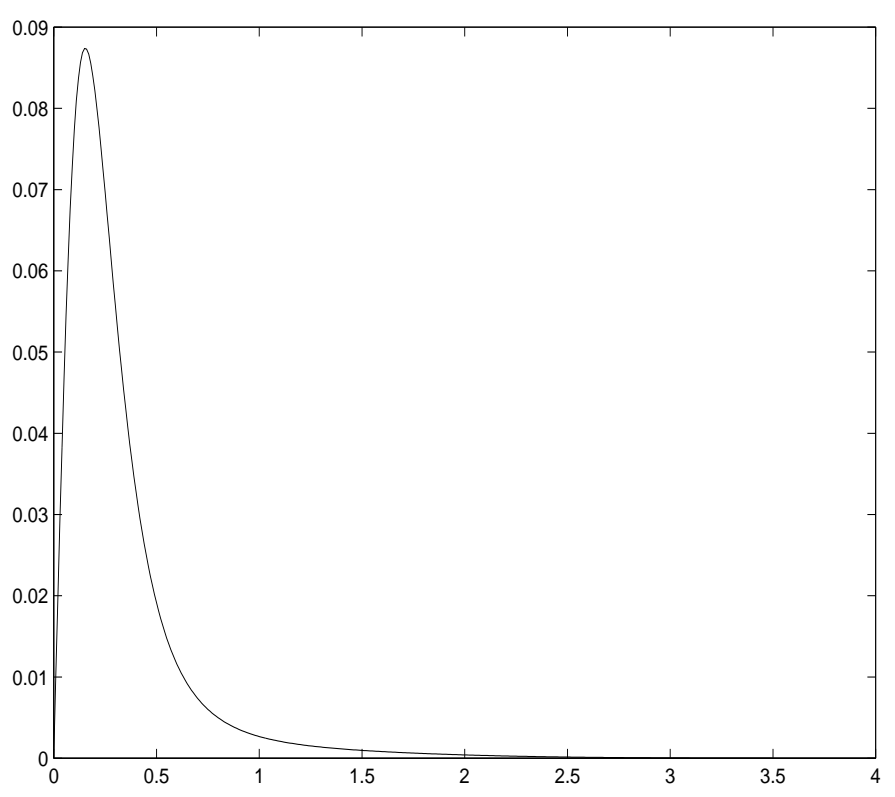

Figure 1: The integrand for the funnel potential for $x_{o}^{6}=2$.

The integral is clearly dominated by the contribution coming from $\sigma \approx 0$, that is, the potential at this observation point is dominated by the contribution from the D5 brane. Decreasing $x_{o}^{6}=0.8$, the integrand behaviour is shown in figure 2 . 


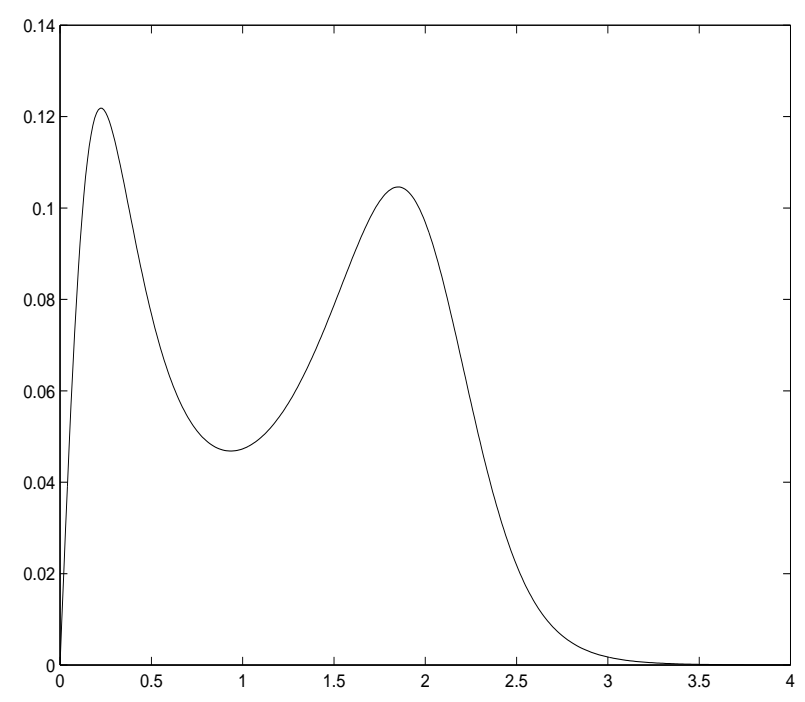

Figure 2: The integrand for the funnel potential for $x_{o}^{6}=0.8$.

We now have two significant contributions - a D5 brane contribution at $\sigma \approx 0$ and a contribution from $\sigma \approx 2$ corresponding to that portion of the funnel closest to the observer, which for large $\sigma_{o}$ looks like a D-string contribution. This behaviour is easy to understand on physical grounds - as we move closer to the funnel, the local geometry has a bigger effect. Decreasing $x_{o}^{6}=0.1$, the integrand behaviour is shown in figure 3 .

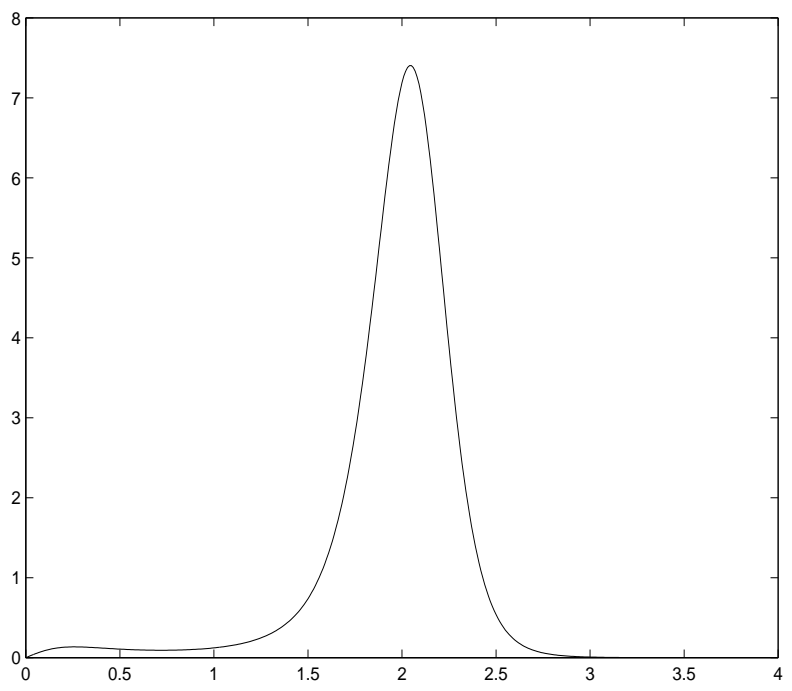

Figure 3: The integrand for the funnel potential for $x_{o}^{6}=0.1$. 
The integrand is now dominated by a D-string contribution - the contribution from the D5 brane at $\sigma \approx 0$ is negligible. Assuming the funnel reproduces the gravitational potential in this third region, we have a convincing explanation of why the fluctuations described by the funnel are the same for both the D $\perp \perp \mathrm{D} 1$ and the $\mathrm{D} 5 \perp \mathrm{D} 1$ systems: only the geometry of the funnel for large $\sigma$ is relevant, and this is the same for both funnels. A comment is in order. In our plots the integrand peaks at $\sigma=2=\sigma_{o}$. We chose this value of $\sigma_{o}$ to produce plots which clearly illustrate our point. In the computation of small fluctuations which can be compared to a test string one would take $\sigma_{o}>>1$ large.

We have not yet discussed what we mean by a "small fluctuation". We have not pursued a suitable definition of what a "small" fluctuation in this Newtonian toy model is. Rather, we have been content to show that there is a choice for the scaling of the fluctuation that (i) ensures that the funnel predictions are reproduced and (ii) for which the fluctuations are small. By reproducing the funnel predictions, we mean we find a potential with a $\frac{1}{\sigma_{o}^{4}}$ dependence. Making the ansatz

$$
x^{d+1} \propto \sigma_{o}^{-\frac{1}{n}},
$$

we find that with $n=\frac{5}{13}$ for the D3 $\perp \mathrm{D} 1$ system and with $n=\frac{7}{13}$ for the D5 $\perp \mathrm{D} 1$ system, the a $\frac{1}{\sigma_{o}^{4}}$ dependence for the potential is obtained. To obtain these values we have performed a steepest descent evaluation of the potential assuming that $\sigma_{o}$ is large. The integrand has a peak at $\sigma \approx \sigma_{o}$. It is pleasing that the fluctuations are indeed small in the large $\sigma_{o}$ region we focus on, showing some degree of self consistency.

To summarize, our toy model shows that the background corresponding to a stack of $n$ D5 branes might not be the appropriate background against which to compare the funnel results. Further, we have shown that, for a very specific assumption about the $\sigma_{o}$ dependence of the fluctuations, the gravitational results and the funnel results are consistent. It would be nice to perform a more complete analysis in supergravity, including a careful independent determination of the scaling of $x^{d+1}$ with $\sigma_{o}$, to confirm that the two are indeed consistent.

\section{Summary}

In this article we have considered the non-commutative fuzzy funnel geometries that provide a description of a D-string ending on a D3, D5 or D7 brane. In particular, we have focused on small fluctuations about the leading funnel geometry. A numerical treatment of these small fluctuations has been given. The connection between zero 
modes and symmetries of fuzzy sphere geometries has been discussed. This connection is particularly transparent for the $\mathrm{D} 1 \perp \mathrm{D} 5$ system with $n=1$. The comparison of the linearized fluctuations of the full Born-Infeld action and the description of these fluctuations in supergravity has been considered in the framework of a Newtonian toy model. An explanation of previous disagreements between the two descriptions has been suggested.

There are a number of ways in which this work can be extended. The connection between zero modes and symmetries of the fuzzy sphere deserves to be studied further. This will involve a careful study of the geometry of the fuzzy sphere. The results of [20] seem to provide a possible approach for this study. Another interesting direction which could be followed would involve improving upon our Newtonian toy model of the funnel. The construction of a supergravity model of the funnel as well as a demonstration that the D-string description agrees with the supergravity description would be satisfying.

Acknowledgements: We would like to thank Sanjaye Ramgoolam for very pleasant and helpful discussions. We also thank Jeff Murugan and Sanjaye Ramgoolam for comments on the manuscript. This work is supported by NRF grant number Gun 2047219.

\section{References}

[1] N.R. Constable, R.C. Myers and O. Tafjord, "Noncommutative bion core", Phys. Rev. D61 106009 (2000) hep-th/9911136.

[2] N.R. Constable, R.C. Myers and O. Tafjord, "Non-abelian brane intersections", JHEP 0106:023 (2001) hep-th/0102080.

[3] D. Kabat and W. Taylor, "Spherical Membranes in Matrix Theory,," Adv. Theor. Math. Phys. 2181 (1998), hep-th/9711078; Soo-Jong Rey, "Gravitating M(atrix) Q-Balls, hep-th/9711081; J. Castelino, S. Lee and W. Taylor, "Longitudinal Five-Branes as Four Spheres in Matrix Theory," Nucl. Phys. B256 334 (1998), hep-th/9712105.

[4] P. Cook, R. de Mello Koch and J. Murugan, "Non-Abelian BIonic brane-intersections," Phys. Rev. D68 126007 (2001), hep-th/0306250.

[5] Y. Kimura, "Nonabelian gauge field and dual description of fuzzy sphere," hep-th/0402044;

J.L. Karczmarek, "Multicore Noncommutative Bions," hep-th/0410233;

A. Basu and J.A. Harvey, "The M2-M5 Brane System and a Generaized Nahm's Equation," hep-th/0412310; 
D.S. Berman and Neil P. Copland, "Five-brane Calibrations and Fuzzy Funnels," hep-th/0504044;

R. Bhattacharyya, "A Short Note on Multi-bion Solutions, hep-th/0505103;

S. Thomas and J. Ward, "Fuzzy Sphere Dynamics and Non-Abelian DBI in Curved Backgrounds," hep-th/0508085.

[6] J. Maldacena and A. Strominger, "AdS3 Black Holes and a Stringy Exclusion Principle," JHEP 9812013 (2002), hep-th/9804085;

A. Jevicki and S. Ramgoolam, "Noncommutative gravity from the AdS/CFT Correspondence," JHEP 9904032 (1999), hep-th/9902059;

Pei-Ming Ho, Sanjaye Ramgoolam and Radu Tatar, "Quantum Spacetimes and Finite $N$ Effects in 4D Super Yang-Mills Theories," Nucl. Phys. B573 364-376 (2000), hep-th/9907145;

A. Jevicki, M. Mihailescu, S. Ramgoolam, "Noncommutative Spheres and the AdS/CFT Correspondence," JHEP 0010008 (2000), hep-th/0006239;

A. Jevicki, M. Mihailescu and S. Ramgoolam, "Hidden Classical Symmetry in Quantum Spaces at Roots of Unity: From Q Sphere to Fuzzy Sphere", hep-th/0008186; P.M. Ho and M. Li, "Fuzzy Spheres in AdS/CFT Correspondence and Holography from Noncommutativity," Nucl. Phys. B596 259 (2001), hep-th/0004072;

M. Berkooz and H. Verlinde, "Matrix Theory, AdS/CFT and Higgs-Coulomb Equivalence," JHEP 9912037 (1999), hep-th/9907100;

G. Bonelli, "Matrix Strings in PP Wave Backgrounds from Deformed Super Yang-Mills Theory," JHEP 0208:022 (2002), hep-th/0205213.

[7] P. Collins and R. Tucker, "Classical and Quantum Mechanics of Free Relativistic Membranes," Mucl. Phys. B112 (1976) 150;

D. Kabat and W. Taylor, "Spherical Membranes in Matrix Theory," Adv. Theor. Math. Phys. 2 (1998) 181, hep-th/9711078;

S. Ramgoolam, S. Spence and S. Thomas, "Resolving Brane Collapse with 1/N Corrections in non-Abelian DBI," Nucl. Phys. B703 (2004) 236, hep-th/0405256;

C. Papgeorgakis and S. Ramgoolam, "Large-small dualities between periodic collapsing/expanding branes and brane funnels," hep-th/0504157.

[8] L. Susskind, "Some Speculations about Black Hole Entropy in String Theory", hep-th/9309145;

A. Sen, "Black Hole Solutions in Heterotic String Theory on a Torus," Nucl. Phys. B440 421-440, (1995) hep-th/9411187;

A. Sen, "Extremal Black Holes and Elementary String States," Mod. Phys. Lett. A10 2081-2094, (1995) hep-th/9504147;

A. Strominger and C. Vafa, "Microscopic Origin of the Bekenstein-Hawking Entropy," Phys. Lett. B379 99-104 (1996) hep-th/9601029;

C.G. Callan and J.M. Maldacena, "D-brane approach to Black Hole Quantum Mechanics," Nucl. Phys. B472 591-610 (1996) hep-th/9602043. 
[9] S.R. Das and S.D. Mathur, "Comparing Decay Rates for Black Holes and D-branes," Nucl. Phys. B478 561-576 (1996) hep-th/9606185;

S.R. Das and S.D. Mathur, "Interactions Involving D-branes," Nucl. Phys. B482 153-172 (1996) hep-th/9607149;

A. Dhar, G. Mandal and S.R. Wadia, Absorption versus Decay of Black Holes in String Theory and T Symmetry," Phys. Lett. B388 51-59 (1996) hep-th/9605234;

J.M. Maldacena and A. Strominger, "Black Hole Grey Body Factors and D-branes Spectroscopy," Phys. Rev. D55 861-870 (1997) hep-th/9609026.

[10] S. Ramgoolam, "On Spherical Harmonics for Fuzzy Spheres in Diverse Dimensions," Nucl. Phys. B610 (2001) 461, hep-th/0105006;

S. Ramgoolam, "Higher dimensional geometries related to Fuzzy odd-dimensional spheres," hep-th/0207111.

[11] Pei-Ming Ho and Sanjaye Ramgoolam, "Higher Dimensional Geometries from Matrix Brane Constructions," hep-th/0111278.

[12] R.C. Myers, "Dielectric-Branes", JHEP 9912, 022 (1999), hep-th/9910053.

[13] A.A. Tseytlin, Born-Infeld Action, Supersymmetry and String Theory, in The Many Faces of the Superworld, ed. M. Shifman (World Scientific, 2000), hep-th/9908105.

[14] A.A. Tseytlin, "On Non-Abelian generalization of Born-Infeld action in String Theory", Nucl.Phys.B501, 41 (1997), hep-th/9701125.

[15] Y. Kimura, "On Higher Dimensional Fuzzy Spherical Branes," hep-th/0301055; T. Azuma and M. Bagnoud, "Curved space classical solutions of a massive supermatrix model," hep-th/0209057.

[16] K.S. Gupta and S.G. Rajeev, "Renormalization in Quantum Mechanics," Phys. Rev. D48 5940 (1993) hep-th/9305052.

[17] A.P. Polychronakos, Les Houches Lectures, 1998, hep-th/9902157;

H.E. Camblong, L.N. Epele, H. Fanchiotti and C.A.G.Canal, "Renormlaization of the Inverse Square Potential," Phys. Rev. Lett. 851593 (2000), hep-th/0003014;

B. Basu-Mallick and K.S. Gupta, "Bound States in One-Dimensional Quantum N-Body Systems with Inverse Square Interaction," hep-th/0109022.

[18] S. Lee, A. Peet and L.Thorlacius, "Brane-Waves and Strings," Nucl. Phys. B514 (1998) 161, hep-th/9710097;

D. Kastor and J. Traschen, "Dynamics of the DBI Spike Soliton," Phys. Rev. D61 (2000), 024034, hep-th/9906237.

[19] Soo-Jong Rey and Jung-Tay Yee, "BPS Dynamics of Triple $(p, q)$ String Junction," Nucl. Phys. B526 (1998) 229, hep-th/9711202;

Soo-Jong Rey and Jung-Tay Yee, "Macroscopic Strings as Heavy Quarks: Large N Gauge Theory and anti-de Sitter Supergravity," Eur. Phys. J. C22 (2001) 379, hep-th/9803001. 
[20] S. Ramgoolam, "Towards gauge theory for a class of commutative and non-associative fuzzy spaces," hep-th/0310153. 